\title{
CONCERNING A SET OF POSTULATES FOR PLANE ANALYSIS
}

\section{SITUS*}

BY

\author{
ROBERT L. MOORE
}

My paper On the foundations of plane analysis situs $\dagger$ contains three sets of postulates, $\Sigma_{1}, \Sigma_{2}$, and $\Sigma_{3}$, expressed in terms of the undefined notions point and region. In the present paper I will show that every space $S$ that satisfies $\Sigma_{1}$ or $\Sigma_{2}$ is a number plane, that is to say there exists, between $S$ and a twodimensional euclidean space $S^{\prime}$, a one-to-one correspondence that preserves limits. $\ddagger$ This signifies that if $P$ is a point and $M$ is a point-set in $S$, and $P^{\prime}$ and $M^{\prime}$ are the corresponding point and point-set in $S^{\prime}$, then $P$ is a limit point of $M$ in the sense defined on page 132 of the above mentioned paper if, and only if, $P^{\prime}$ is a limit point of $M^{\prime}$ in the sense that every circle in $S^{\prime}$ that encloses $P^{\prime}$ encloses also a point of $M^{\prime}$ distinct from $P^{\prime}$. It follows that $\Sigma_{1}$ and $\Sigma_{2}$ are both categorical with respect to $\S$ point and limit point as defined on page 132. Moreover between every space $S$, satisfying $\Sigma_{1}$, and a two-dimensional euclidean space $S^{\prime}$ there exists a one-to-one correspondence preserving point and region if in $S^{\prime}$ the term region is interpreted to mean Jordan region. That is to say if the set of points $M$ is a region in $S$ then the set $M^{\prime}$ of corresponding points in $S^{\prime}$ is the interior of a simple closed curve and conversely. Thus $\Sigma_{1}$ is absolutely $\|$ categorical. The system $\Sigma_{2}$ is satisfied if in ordinary euclidean space of two dimensions the term region is interpreted as signifying Jordan region. It is however also satisfied if in such a space region is so interpreted

* Presented to the Society, December 27, 1917.

† These Transactions, vol. 17 (1916), pp. 131-164. Hereafter this paper will be referred to as Foundations.

$\ddagger$ This is not true of $\Sigma_{3}$. Indeed there exist spaces satisfying $\Sigma_{3}$ that are neither metrical, descriptive, nor separable. Cf. Foundations, pp. 131, 132, and 162. In the paper referred to in the A nnals of Mathe matics, vol. 16 (1915), p. 131, in the statement of Condition I, " $\left|y_{k}^{\prime}-y_{k}^{\prime \prime}\right|<\epsilon$ " is to be omitted in (1) and inserted (with the conjunction "and" prefixed) immediately after " $\left|x_{k}^{\prime}-x_{k}^{\prime \prime}\right|=0$ " in (2). For certain results concerning the relation between Fréchet's Calcul Fonctionnel and spaces that satisfy $\Sigma_{1}$ or $\Sigma_{2} \mathrm{cf}$. E. W. Chittenden, Bulletin of the American Mathematical Society, vol. 23 (1917), p. 390.

§ Cf. my paper The linear continuum in terms of point and limit, A n a ls of $\mathrm{Mat}$ he m a ti c s, loc. cit., p. 127.

\|l Loc. cit., p. 127. 
as to apply to every bounded, connected set of points $R$ of connected exterior such that (1) every point of $R$ lies in the interior of some triangle that is contained in $R,(2)$ every point of the boundary of $R$ is a limit point of the exterior of $R$. Thus, though categorical with respect to point and limit point, $\Sigma_{2}$ is not absolutely categorical.

In my proof that $\Sigma_{1}$ is categorical I will make use of the following theorems.

TheOREM A. If $L$ is a simple closed curve and $M$ and $N$ are two closed point-sets with no point in common, there does not exist an infinite set of arcs $G$ such that (1) each arc of the set $G$ is a subset of $L,(2)$ each arc of $G$ contains a point of $M$ and also a point of $N$, (3) no two arcs of $G$ have a segment in common.

Proof. * Suppose there exists an infinite set of such arcs $A_{1} B_{1}, A_{2} B_{2}, \ldots$ where $A_{1}, A_{2}, \cdots$ are points of $M$ and $B_{1}, B_{2}, \cdots$ are points of $N$. Then there exist points $A$ and $B$ and an infinite subsequence $A_{n_{1}} B_{n_{1}}, A_{n_{2}} B_{n_{2}}, \ldots$ such that $A$ is a sequential limit point of $A_{n_{1}}, A_{n_{2}}, \cdots$ and $B$ is a sequential limit point of $B_{n_{1}}, B_{n_{2}}, \cdots$. There exist on $L$ four points $C, D, E$, and $F$ in the order $E C A D F B$. There exists a positive number $\delta$ such that if $m>\delta$ then $A_{n_{m}}$ and $B_{n_{m}}$ are on the segments $C A D$ and $E B F$ respectively of the curve $L$. It follows that if $p, q$, and $r$ are three distinct positive integers greater than $\delta$ then two of the $\operatorname{arcs} A_{n_{p}} B_{n_{p}}, A_{n_{q}} B_{n_{q}}, A_{n_{r}} B_{n_{r}}$ have in common either a segment from $C$ to $E$ or a segment from $D$ to $F$. But this is contrary to supposition.

Theorem B. If $J$ and $L$ are two simple closed curves and $A$ and $B$ are two distinct points of $J$ each of which is either not on $L$ at all or on some segment* that is common to $J$ and $L$, then there exists an arc from $A$ to $B$ that lies, except for its endpoints, entirely within $J$ and has not more than a finite number of points in common with $L$.f

Proof. By Theorem $40 \S$ there exists an arc $A B$ that lies, except for its endpoints, entirely within $J$. If the segment $A B$ and the closed curve $L$ have points in common let $M$ denote the set of all such common points. In view

* Cf. the proof of Theorem 37 on pages 152 and 153 of Foundations.

† If $X$ and $Y$ are two points of a closed curve $J$, there are just two arcs that lie wholly on $J$ and have $A$ and $B$ as endpoints. Each of these arcs is called an interval of $J$. An interval minus its endpoints is a segment.

$\ddagger$ The following example shows that this theorem would not be true if no restriction were imposed on the relative positions of $A, B, J$, and $L$ other than merely that $A$ and $B$ should lie somewhere on $J$.

Example. Let $J$ denote the perimeter of the triangle whose vertices are $(0,0),(2,1)$, and $(2,-1)$. Let $L$ be the closed curve bounded by the arc from $(0,0)$ to $(1 / \pi, 0)$ on the curve $y=x \sin (1 / x)$, and straight line intervals from $(1 / \pi, 0)$ to $(2,-2)$, from $(2,-2)$ to $(0,-2)$ and from $(0,-2)$ to $(0,0)$ respectively. Let $A$ and $B$ be the points $(0,0)$ and $(2,1 / 2)$ respectively. Clearly there is no arc from $A$ to $B$ that lies, except for its endpoints, entirely within $J$ and has only a finite number of points in common with $L$.

$\S$ Numbered theorems are theorems in my paper On the foundations of plane analysis situs, loc. cit. 
of the conditions of our hypothesis it is clear that there exists a point $A_{1}$ which is the first* point that the segment $A B$ has in common with $L$. If there exist any points of $M$ that can be joined to $A_{1}$ by an arc of $L$, that lies entirely within $J$ then there must be a last such point. For otherwise there would exist on $A B$ a point $X$ which cannot be joined to $A_{1}$ by an arc of $L$ lying entirely within $J$ but which is a limit point of a set of points $K$ that lie on $A B$ and each of which can be so joined to $A_{1}$. But in this case there would exist on $L$ a segment $Y X Z$ containing $X$ and lying entirely within $J$. The segment $Y X Z$ must $\dagger$ contain at least one point $P$ of the set $K$. But $P$ could be joined to $A_{1}$ by an arc $A_{1} P$ of $L$ that lies entirely within $J$, and the point-set $A_{1} P+Y X Z$ would be an arc of $L$ lying entirely within $J$. Thus the supposition that there exists no last point that can be properly $\ddagger$ joined to $A_{1}$ leads to a contradiction. Let $B_{1}$ denote the last point on $A B$ that can be properly joined to $A_{1}$. By an argument similar to the above proof of the non-existence of $X$ it can be shown that $B_{1}$ cannot be a limit point of a set of points of $M$ no one of which can be properly joined to $A_{1}$. But $B_{1}$ is the last point that can be properly joined to $A_{1}$. It follows that on $A B$ there must be a point $W$, following $B_{1}$, such that the segment $B_{1} W$ of the arc $A B$ is entirely free of points of $L$. But $L$ is a closed set of points. It follows that if there are on the segment $A B$ any points of $L$ that follow $B_{1}$ then there is a first such point, $A_{2}$. There exists $\S$ a point $B_{2}$ which is the last point on $A B$ that can be properly joined to $A_{2}$. If there are any points of $M$ after $B_{2}$ there is $\|$ a point $A_{3}$ which is the first such point. Continue this process. There must exist only a finite number of the points $A_{1}, A_{2}, A_{3}, \ldots$. For suppose there were infinitely many such points. Then there must be infinitely many points $B_{1}, B_{2}, B_{3}, \cdots$. Suppose $n$ is a positive integer. The points $B_{n}$ and $A_{n+1}$ are the extremities of two arcs of $L$. Let $B_{n} A_{n+1}$ denote one of these arcs. The arc $B_{n} A_{n+1}$ does not lie wholly within $J$. Hence there exists a point $X_{n}$ which is the first point that it has in common with $J$. Let $B_{n} X_{n}$ denote the arc from $B_{n}$ to $X_{n}$ on the $\operatorname{arc} B_{n} A_{n+1}$. Let $i$ and $j$ be two positive integers. If the segments $B_{i} X_{i}$ and $B_{i+j} X_{i+j}$ had a point in common then $B_{i+j}$ could be properly joined to $B_{i}$. But $B_{i}$ can be properly joined to $A_{i}$. Hence $B_{i+j}$ could be properly joined to $A_{i}$. But $B_{i}$ is the last point of $M$ that can be properly joined to $A_{i}$ and $B_{i+j}$ follows $B_{i}$. It follows that the segments $B_{i} X_{i}$ and $B_{i+j} X_{i+j}$ have no point in common. Thus if there

* Cf. Foundations, p. 139. Hereafter in this paper, unless the contrary is indicated, by "first point" and "last point" will be meant first and last point respectively in the order from $A$ to $B$ on the $\operatorname{arc} A B$.

$\dagger$ Loc. cit., p. 139.

$\ddagger$ The phrase "can be properly joined to $A_{1}$ " is an abbreviation for "is identical with $A_{1}$ or can be joined to $A_{1}$ by an arc of $L$ that lies entirely within $J$."

$\S$ Cf. the above proof of the existence of $B_{1}$.

\|Cf. the above proof of the existence of $A_{2}$. 
are infinitely many of the points $A_{1}, A_{2}, A_{3}, \cdots$ then there are infinitely many arcs, all lying on $L$, such that no two of them have, in common, any point unless possibly a common endpoint, and such that each of them has one endpoint on $J$ and the other on the interval $A_{1} O$ of the arc $A B$ where $O$ is the last point before $B$ that $A B$ has in common with $L$. But by Theorem $A$ a simple closed curve cannot contain an infinite set of arcs of this type. It follows that there must exist only a finite number $m$ of the points $A_{1}, A_{2}$, $A_{3}, \cdots$. It is clear that $O$ is either $A_{m}$ or $B_{m}$. For every positive integer $n$, less than $m$, if $A_{n} \neq B_{n}, A_{n}$ can be joined to $B_{n}$ by an $\operatorname{arc} A_{n} Z_{n} B_{n}$ that belongs to $L$ and lies entirely within $J$. It is easy to see, with the aid of Theorems 43 and 40, that there exists an arc $A_{n} \bar{Z}_{n} B_{n}$ that lies entirely within $J$ and, except for its endpoints, is also entirely within $L$. Consider the pointset $\tau$ composed of $A A_{1}+\left(A_{1} \bar{Z} B_{1}\right)+B_{1} A_{2}+\left(A_{2} \bar{Z}_{2} B_{2}\right)+B_{2} A_{3}+\cdots$ $+B_{m-1} A_{m}+\left(A_{m} \bar{Z}_{m} B_{m}\right)+O B$ where $A A_{1}, B_{1} A_{2}, B_{2} A_{3}, \cdots B_{m-1} A_{m}$ and $O B$ are intervals of the arc $A B$ and for each $i(1 \leqq i \leqq m)$ " $\left(A_{i} \bar{Z}_{i} B_{i}\right)$ " denotes the arc $A_{i} \bar{Z} B_{i}$ or the point $A_{i}$ according as $B_{i}$ is not or is identical with $A_{i}$. The point-set $B_{n} A_{n+1}(1 \leqq n \leqq m-1)$ contains in common with $L$ no points except $B_{n}$ and $A_{n+1}$. In view of these considerations it is clear that the point-set $\tau$ contains, as a subset, an arc from $A$ to $B$ that lies, except for its endpoints, entirely within $J$ and has not more than $2(m+1)$ points in common with $L$. The truth of Theorem $B$ is therefore established.

Theorem C. If the closed curve $g$ has only a finite number of points in common with the closed curve $A B C D A$ and does not contain $A, B, C$, or $D$ then the interior of $A B C D A$ can be divided by a double ruling* (such that the arcs of one of its two single rulings are parallel to $A B$ and $C D$ and those of the other are parallel to $A D$ and $B C$ ) into subdivisions such that the interior of each one of them is either wholly within or wholly without $g$.

Indication of proof. There exists a finite set $K$ of arcs of $g$ each of which lies entirely within $A B C D A$ except that its endpoints are on $A B C D A$ and such that every point of $g$ that is within $A B C D A$ lies on an arc of $K$. If $t$ is an arc of the set $K$, either (1) $t$ has both endpoints on the same side $\dagger$ of

\footnotetext{
* If, on the closed curve $A B C D A, X_{1}, X_{2}, \cdots X_{n-1}, X_{n}, Y_{1}, Y_{2}, \cdots Y_{n-1}, Y_{n}, X_{n}^{\prime}$, $X_{n-1}^{\prime}, \cdots X_{2}^{\prime} X_{1}^{\prime}, Y_{n}^{\prime}, Y_{n-1}^{\prime}, \cdots Y_{2}^{\prime} Y_{1}^{\prime}$ are points in the order $A X_{1} X_{2} \cdots X_{n-1} X_{n} B Y_{1} Y_{2}$ $\cdots Y_{n-1} Y_{n} C X_{n}^{\prime} X_{n-1}^{\prime} \cdots X_{2}^{\prime} X_{1}^{\prime} D Y_{n}^{\prime} Y_{n-1}^{\prime} \cdots Y_{2}^{\prime} Y_{1}^{\prime} A$ and $X_{1} X_{1}^{\prime}, X_{2} X_{2}^{\prime}, \cdots X_{n} X_{n}^{\prime}$, $Y_{1} Y_{1}^{\prime}, Y_{2} Y_{2}^{\prime}, \cdots Y_{n} Y_{n}^{\prime}$ are arcs which, except for their endpoints, lie entirely within $A B C D A$, and finally, for every $i, j(i \leqq i \leqq n, 1 \leqq j \leqq n), X_{i} X_{i}^{\prime}$ has just one point in common with $Y_{j} Y_{j}^{\prime}$ and no point in common with $X_{j} X_{j}^{\prime}$ (unless $i=j$ ), then these two sets of arcs are said to constitute a double ruling of the interior of $A B C D A$ (or merely a double ruling of $A B C D A$ ). The $\operatorname{arcs} X_{1} X_{1}^{\prime}, X_{2} X_{2}^{\prime}, \cdots X_{n} X_{n}^{\prime}$ are said to be parallel to $B C$ and to $A D$ and the $\operatorname{arcs} Y_{1} Y_{1}^{\prime}, Y_{2} Y_{2}^{\prime}, \cdots Y_{n} Y_{n}^{\prime}$ are said to be parallel to $A B$ and to $C D$. Each of these two sets of arcs is a single ruling of the double ruling formed by the two sets combined. Of course the word parallel is not used here in the same sense as in ordinary plane geometry.

$\dagger$ The segments $A B, B C, C D$, and $D A$ of the closed curve $A B C D A$ are called the sides of $A B C D A$. By the interval $A B$ of $J$ will be meant that one which does not contain $C$.
} 
$A B C D A$, (2) $t$ has one endpoint on one side, and the other one on an adjacent side, of $A B C D A$, or (3) $t$ has one endpoint on one side, and the other one on an opposite side, of $A B C D A$. Let us suppose, for example, that there are four $\operatorname{arcs} A_{1} B_{1} C_{1} D_{1} E_{1}, A_{2} B_{2} C_{2} D_{2} E_{2}, A_{3} B_{3} C_{3} D_{3} E_{3}$, and $A_{4} B_{4} C_{4} D_{4} E_{4}$, of type 1, two arcs $A_{5} B_{5} E_{5}$ and $A_{6} B_{6} E_{6}$, of type 2 , and one arc $A_{7} E_{7}$ of type 3 , situated as indicated in Fig. 1. In this figure a double ruling $\tau$, satisfying the conditions of Theorem $\mathrm{C}$, is represented. This indication of how a ruling may be constructed in the case of this typical example is given instead of a tedious formal proof covering all possible cases. Here the arcs of $\tau$ that are parallel to $A B$ are $K_{1} D_{1} C_{1} B_{1} L_{1}, K_{2} D_{2}$ $C_{2} B_{2} L_{2}, A_{4} B_{4} L_{3}, A_{3} B_{3} L_{4}, E_{3} D_{3} L_{5}, E_{4}$ $D_{4} L_{6}, E_{7} A_{7}, K_{3} B_{6} A_{6}$, and $K_{4} B_{5} A_{5}$. Those parallel to $B C$ are $H_{1} B_{3} C_{3} D_{3} M_{6}$, $H_{2} B_{4} C_{4} D_{4} M_{5}, E_{2} D_{2} M_{4}, E_{1} D_{1} M_{3}, A_{1}$ $B_{1} M_{2}, A_{2} B_{2} M_{1}, H_{3} B_{6} E_{6}$, and $H_{4} B_{5} E_{5}$.

Theorem D. If $A B C D A$ is a closed curve and $G$ is a set of closed curves and each point on or within $A B C D A$ is within some curve of the set $G$ then the interior of $A B C D A$

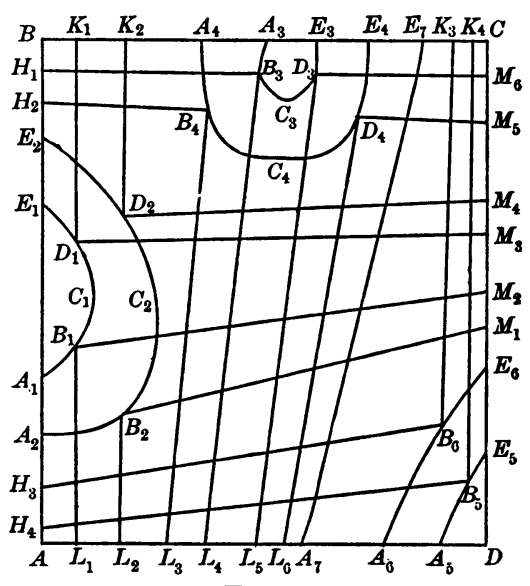

Fig. 1 can be divided by a double ruling (such that the arcs of one of its single rulings are parallel to $A B$ and $C D$ and those of the other are parallel to $A D$ and $B C$ ) into subdivisions each of which is within some curve of the set $G$.

Proof. There exists a finite set $\bar{G}$ of closed curves $g_{1}, g_{2}, \cdots g_{m}$ such that every one of them is a curve of the set $G$ and such that every point on or within $J^{*}$ is within one of them. Every point of the interval $A B$ of $J$ is within some curve of the set $\bar{G}$. It follows that for each point of $A B$ there is a segment of $J$, containing that point, lying wholly within some curve of $\bar{G}$ and not containing any side of $A B C D A$. There exists a finite subset, $Q$, of these segments such that every point of $A B$ is on some segment of $Q$. The set $Q$ contains as a subset a set of segments $A_{1} B_{1}, A_{2} B_{2}, \cdots A_{n} B_{n}$ with endpoints in the order $A_{1} A A_{2} B_{1} A_{3} B_{2} A_{4} B_{3} A_{5} B_{4} \cdots A_{n} B_{n-1} B B_{n}$. There exists a set of points $E_{1}, E_{2}, \cdots E_{n-1}$ and $\operatorname{arcs} E_{1} E_{1}^{\prime}, E_{2} E_{2}^{\prime}, \cdots E_{n-1} E_{n-1}^{\prime}$ such that, for every $i$ and $j(1 \leqq i \leqq n-1,1 \leqq j \leqq n-1, i \neq j)$, (1) $E_{i}$ is in the order $A A_{i+1} E_{i} B_{i} B$, (2) $E_{i} E_{i}^{\prime}$ lies within every region of the set $\bar{G}$ that contains $E_{i}$, (3) every point of $E_{i} E_{i}^{\prime}$ except $E_{i}$ is within $J$, (4) $E_{i} E_{i}^{\prime}$ and $E_{j} E_{j}^{\prime}$ have no point in common, (5) not every point of the segment $E_{i} E_{i}^{\prime}$ is on $g_{1}$. There exists a set of curves $\bar{g}_{1}, \bar{g}_{2}, \cdots g_{n}$, belonging to the set $\bar{G}$, such that $A_{i} B_{i}(1 \leqq i \leqq n)$ is entirely within $\bar{g}_{i}$. Within $\bar{g}_{1}$ (and

* Hereafter the letter $J$ will be used, at times, as an abbreviation for $A B C D A$. 
within $J$ except for the point $A_{1}$ ) there is* an arc $A_{1} E_{1}^{\prime}$ that has in common with $E_{1} E_{1}^{\prime}$ only the point $E_{1}^{\prime}$. Within $\bar{g}_{2}$ and $J$ there is an arc $E_{1}^{\prime} E_{2}^{\prime}$ that has in common with $E_{1} E_{1}^{\prime}$ only the point $E_{1}^{\prime}$ and has in common with $E_{2} E_{2}^{\prime}$ only the point $E_{2}^{\prime}$ and in common with $A_{1} E_{1}^{\prime}$ only the point $E_{1}^{\prime}$. There exists a set of arcs $A_{1} E_{1}^{\prime}, E_{1}^{\prime} E_{2}^{\prime}, E_{2}^{\prime} E_{3}^{\prime}, \cdots E_{n-1}^{\prime} B_{n}$ such that (1) $E_{i}^{\prime} E_{i+1}^{\prime}$ is within $\bar{g}_{i+1}$ and $J(1 \leqq i \leqq n-2), E_{n-1}^{\prime} B_{n}$ is within $\bar{g}_{n}$ and, except for the point $B_{n}$, within $J,(2)$ no two non-consecutive arcs of this set have any point in common and two consecutive ones have only one endpoint in common. It easily follows that there exist (Fig. 2) (1) on $A B C D A, 4 n$ points in the order $A E_{1} E_{2} \cdots E_{n} B F_{1} F_{2} \cdots F_{n} C L_{1} L_{2} \cdots L_{n} D M_{1} M_{2} \cdots M_{n} A$, (2) within

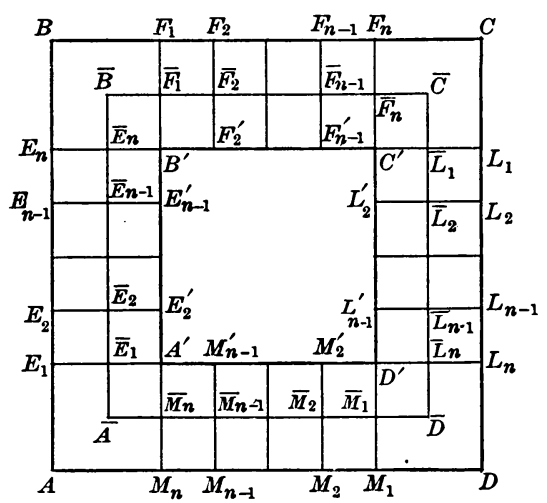

FIG. 2

$A B C D A$, a closed curve $J^{\prime}$ containing $4(n-1)$ points in the order $A^{\prime} E_{2}^{\prime} E_{3}^{\prime}$ $\cdots E_{n-1}^{\prime} B^{\prime} F_{2}^{\prime} F_{3}^{\prime} \cdots F_{n-1}^{\prime} C^{\prime} L_{2}^{\prime} L_{3}^{\prime} \cdots L_{n-1}^{\prime} D^{\prime} M_{2}^{\prime} M_{3}^{\prime} \cdots M_{n-1}^{\prime} A^{\prime}$ ， (3) arcs $A^{\prime} \bar{E}_{1} E_{1}, E_{2}^{\prime} \bar{E}_{2} E_{2}, E_{3}^{\prime} \bar{E}_{3} E_{3}, \cdots, E_{n-1}^{\prime} \bar{E}_{n-1} E_{n-1}, B^{\prime} \bar{E}_{n} E_{n}, B^{\prime} \bar{F}_{1} F_{1}$,

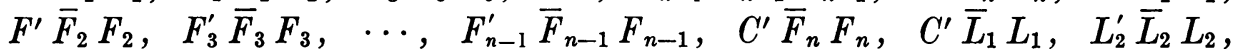
$\cdots L_{n-1}^{\prime} \bar{L}_{n-1} L_{n-1}, D^{\prime} \bar{L}_{n} L_{n}, D^{\prime} \bar{M}_{1} M_{1}, M_{2}^{\prime} \bar{M}_{2} M_{2}, \cdots M_{n-1}^{\prime} \bar{M}_{n-1} M_{n-1}$, $A^{\prime} \bar{M}_{n} M_{n}$ such that every one of these arcs lies, except for its endpoints, entirely between $J^{\prime}$ and $J$ and no two of them have any point in common except that each of the points $A^{\prime}, B^{\prime}, C^{\prime}$, and $D^{\prime}$ is a common endpoint of two of them and such that no one of the points $\bar{E}_{1}, \bar{E}_{2}, \bar{E}_{3}, \ldots \bar{E}_{n-1}, \bar{E}_{n}$, $\bar{F}_{1}, \bar{F}_{2}, \cdots \bar{F}_{n-1}, \bar{F}_{n}, \bar{L}_{1}, \bar{L}_{2}, \bar{L}_{3}, \cdots \bar{L}_{n}, \bar{M}_{1}, \bar{M}_{2}, \bar{M}_{3}, \cdots \bar{M}_{n-1}, \bar{M}_{n}$ is on the curve $g_{1}$; the domain bounded by $J$ and $J^{\prime}$ being divided by these arcs into subdivisions each of which lies within some curve of the set $\bar{G}$. By Theorem $B$ there exists a set, $\alpha$, of arcs $\bar{E}_{i} \bar{E}_{i+1}(1 \leqq i \leqq n-1), \bar{E}_{n} \bar{F}_{1}$, $\bar{F}_{i} \bar{F}_{i+1}(1 \leqq i \leqq n-1), \bar{F}_{n} \bar{L}_{1}, \bar{L}_{i} \bar{L}_{i+1}(1 \leqq i \leqq n-1), \bar{L}_{n} \bar{M}_{1}, \bar{M}_{i} \bar{M}_{i+1}$

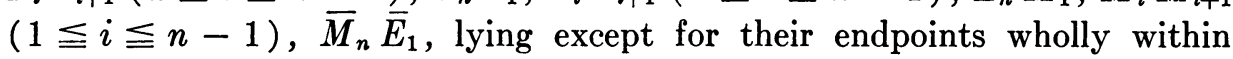
$E_{i} E_{i}^{\prime} E_{i+1}^{\prime} E_{i+1}, E_{n} B^{\prime} F_{1} B, \quad F_{i} F_{i}^{\prime} F_{i+1}^{\prime} F_{i+1}, \quad F_{n} C L_{1} C^{\prime}, L_{i} L_{i}^{\prime} L_{i+1}^{\prime} L_{i+1}$, $L_{n} D^{\prime} M_{1} D, M_{i} M_{i}^{\prime} M_{i+1}^{\prime} M_{i+1}$, and $M_{n} A^{\prime} E_{1} A$ respectively $\dagger$ no one of these

* Make use of Theorems 43 and 40 and the existence of the curve ABTNA described on page 149 of Foundations.

$\dagger$ Here $E_{1}^{\prime}=M_{n}^{\prime}=A^{\prime}, E_{n}^{\prime}=F_{1}^{\prime}=B^{\prime}, F_{n}^{\prime}=L_{1}^{\prime}=C^{\prime}$ and $L_{n}^{\prime}=M_{1}^{\prime}=D^{\prime}$. 
arcs having more than a finite number of points in common with $g_{1}$. There exist, on the segments $\bar{E}_{n} \bar{F}_{1}, \bar{F}_{n} \bar{L}_{1}, \bar{L}_{n} \bar{M}_{1}$, and $\bar{M}_{n} \bar{E}_{1}$ respectively, points $\bar{B}, \bar{C}, \bar{D}$, and $\bar{A}$ that are not on $g_{1}$. The ares of $\alpha$ form a closed curve $\bar{A} \bar{B} \bar{C} \bar{D} \bar{A}$ which will, at times, be called $\bar{J}$. This curve does not have more than a finite number of points in common with $g_{1}$. Furthermore the domain $\omega$ bounded by $\bar{A} \bar{B} \bar{C} \bar{D} \bar{A}$ and $A B C D A$ is subdivided by the $\operatorname{arcs} E_{i} \bar{E}_{i}, F_{i} \bar{F}_{i}$, $L_{i} \bar{L}_{i}, M_{i} \bar{M}_{i}(1 \leqq i \leqq n)$ into subdivisions each of which lies wholly within some curve of the set $\bar{G}$. By Theorem $C$ the interior of $\bar{A} \bar{B} \bar{C} \bar{D} \bar{A}$ may be divided by a double ruling $\bar{\tau}$ (such that the arcs of one of its two single rulings are parallel to $A B$ and $C D$ and those of its other one are parallel to $B C$ and $A D$ ) into subdivisions the interior of each one of which is either entirely within or entirely without $g_{1}$. By the addition of certain arcs having their endpoints on $J$ and $\bar{J}$ and lying, except for their endpoints, entirely between $J$ and $\bar{J}$, it is possible to continue the arcs of $\bar{\tau}$, together with suitably chosen additional arcs, in such a way that there will result a double ruling $\tau_{1}$ of $A B C D A$ such that every arc of the set $\alpha$ and every arc of the double ruling $\bar{\tau}$ will be a portion of some arc of the double ruling $\tau_{1}$. The interior of each subdivision of the ruling $\tau_{1}$ is either within one of the subdivisions into which $\bar{I}^{*}$ is divided by the rulings of $\bar{\tau}$ or within one of the subdivisions into which the domain $\omega$ is divided by the ares of the set $\alpha$. It follows that the interior of each of the subdivisions into which $I$ is divided by $\tau_{1}$ is either wholly within some curve of the set $\bar{G}$ or wholly without $g_{1}$. If any of them are wholly without $g_{1}$ then each such subdivision can itself be divided by a double ruling (the arcs of one single ruling of which are parallel to one side, $\dagger$ and the arcs of the other single ruling of which are parallel to an adjacent side of this subdivision) into subdivisions each of which is either wholly within some curve of the set $\bar{G}$ or wholly without $g_{2}$. The arcs of these rulings may be extended in such a way that there will result a new double ruling $\tau_{2}$ of $A B C D A$ such that each arc of $\tau_{1}$ is also an arc of $\tau_{2}$ and each subdivision formed by $\tau_{2}$ is within some subdivision formed by $\tau_{1}$ and is either wholly without $g_{1}$ and wholly without $g_{2}$ or wholly within some curve of the set $\bar{G}$. It can be shown in a similar way that there exists a double ruling $\tau_{3}$ of $A B C D A$ such that the interior of every subdivision formed by $\tau_{3}$ is either wholly without each of the curves $g_{1}, g_{2}$, and $g_{3}$ or wholly within some curve of the set $\bar{G}$. This process may be continued. It follows that there exists a double ruling $\tau_{n}$ of $A B C D A$ such that every one of the subdivisions into which $\tau_{n}$ divides $A B C D A$ is either wholly without every curve of the set $g_{1}, g_{2}, \cdots, g_{m}$ or wholly within one of them. But by hypothesis every point of the interior of $A B C D A$ is

${ }^{*} I$ and $\bar{I}$ denote the interiors of $J$ and $\bar{J}$ respectively.

$\dagger$ A side of one of the subdivisions into which the double ruling $\tau_{1}$ divides $I$ is a segment of the boundary of that subdivision which lies between two consecutive arcs of one of the single rulings of $\tau_{1}$ and has its endpoints on those ares. 
within some curve of the set $g_{1}, g_{2}, \cdots, g_{m}$. It follows that every subdivision of $I$ formed by the ruling $\tau_{n}$ is wholly within some curve of this set.

Theorem E. If $A B C D A$ is a closed curve there exist two sets of arcs $\alpha_{1}$ and $\alpha_{2}$ such that (1) each arc of $\alpha_{1}$ lies wholly within ABCDA except that its endpoints are on $A B$ and $C D,(2)$ each arc of $\alpha_{2}$ lies wholly within $A B C D A$ except that its endpoints are on $B C$ and $D A$, (3) each point on $A B C D A$, with the exception of $A, B, C$, and $D$, is an endpoint either of just one arc of $\alpha_{1}$ or of just one arc of $\alpha_{2}$, (4) through each point within $A B C D A$ there is just one arc of $\alpha_{1}$ and just one arc of $\alpha_{2}$, (5) each arc of $\alpha_{1}$ has just one point in common with each arc of $\alpha_{2}$.

Proof. For each positive integer $n$ there exists, about each point $P$ of $A B C D A$ plus its interior, a fundamental* region of subscript greater than $n$. For each $n$ let $G_{n}$ denote the set of all such fundamental regions. for all such points $P$. By Theorem $D$ the interior of $A B C D A$ can be divided by a double ruling $\beta_{1}$ into subdivisions each of which is within some region of the set $G_{1}$. Each of these subdivisions can be divided by a double ruling into subdivisions each of which is within some region of the set $G_{2}$, the arcs of this ruling being so chosen that they can be extended in such a way that there will result a double ruling $\beta_{2}$ of $A B C D A$ such that each arc of $\beta_{1}$ is an arc of $\beta_{2}$ and such that each subdivision of $\beta_{2}$ is within some region of the set $G_{2}$. This process may be continued. It follows that there exists an infinite sequence $\beta_{1}, \beta_{2}$, $\beta_{3}, \cdots$ of rulings of $A B C D A$ such that (1) for each $n$, every arc of the ruling $\beta_{n}$ belongs also to the ruling $\beta_{n+1}$, (2) every subdivision of the ruling $\beta_{n}$ is within some fundamental region of subscript more than $n$. Let $\left\{\bar{a}_{2}\right\}$ denote the set of all arcs $\left\{\begin{array}{l}s_{2} \\ a_{1}\end{array}\right\}$ such that $\left\{\begin{array}{l}s_{2} \\ s_{1}\end{array}\right\}$ has one endpoint on $\left\{\begin{array}{l}B C \\ A B\end{array}\right\}$ and the other on $\left\{\begin{array}{l}D A \\ C D\end{array}\right\}$ and belongs to some $\beta_{n}$. If $P$ is a point within or on $A B C D$, distinct from $A, B, C$, and $D$, and not on any arc of the set $\left\{\bar{a}_{a_{1}}\right\}$ it can be proved that there exists through $P$, and with endpoints on $A B C D A$, just one arc that does not intersect any arc of the set $\left\{\frac{\bar{a}_{2}}{\bar{a}_{1}}\right\}$. Let $\left\{\begin{array}{l}a_{2}^{*} \\ a_{1}^{*}\end{array}\right\}$ be the set of all such arcs for all such points $P$. Let $\left\{\begin{array}{l}a_{2} \\ a_{1}\end{array}\right\}$ denote the set of all arcs of the set $\left\{\begin{array}{l}\bar{a}_{2} \\ \bar{a}_{1}\end{array}\right\}$ together with all arcs of the set $\left\{\begin{array}{l}a_{2}^{*} \\ a_{1}^{2}\end{array}\right\}$. The sets $\alpha_{1}$ and $\alpha_{2}$ satisfy the conditions of Theorem E.

Theorem F. There exists a countably infinite sequence of closed curves $J_{1}, J_{2}, J_{3}, \cdots$ such that every point lies within one of them and such that, for every $n, J_{n+1}$ encloses $J_{n}$.

Proof. By Theorem 36 every point is within some closed curve. It follows, with the aid of Theorem 12, that there exists, for each $n$, a finite set $G_{n}$ of closed curves such that every point of the fundamental region $R_{n}$ is within some curve of the set $G_{n}$ and every curve of the set $G_{n}$ encloses a point of $R_{n}$. By Theorem 42 there exists a closed curve $J_{n}^{\prime}$ that encloses the interiors of all

* Cf. Foundations, p. 133. 
the curves of the set $G_{n}$ and therefore encloses $R_{n}$. If $A_{1}^{\prime}$ is a point within $J_{1}^{\prime}$ and $A_{2}^{\prime}$ is a point within $J_{2}^{\prime}$ then by Theorem 15 there exists an $\operatorname{arc} A_{1}^{\prime} A_{2}^{\prime}$. There exists a finite set of curves enclosing $J_{1}^{\prime}+J_{2}^{\prime}+A_{1}^{\prime} A_{2}^{\prime}$. It follows that there exists a closed curve $J_{2}$ enclosing $J_{1}^{\prime}, R_{1}$, and $R_{2}$. Similarly there exists a closed curve $J_{3}$ enclosing $J_{2}, R_{1}, R_{2}$, and $R_{3}$. This process may be continued. It follows that there exists a countably infinite sequence of closed curves $J_{1}, J_{2}, J_{3}, \cdots$ such that, for every $n, J_{n+1}$ encloses $J_{n}, R_{1}, R_{2}, \cdots$, $R_{n}$. But every point lies in some region of the set $R_{1}, R_{2}, R_{3}, \cdots$. Thus the sequence $J_{1}, J_{2}, J_{3}, \cdots$ satisfies the conditions of Theorem F.

Theorem G. There exist two sets $G_{1}$ and $G_{2}$ of open curves such that (1) through each point there is just one curve of $G_{1}$ and just one curve of $G_{2},(2)$ each curve of $G_{1}$ has just one poirt in common with each curve of $G_{2}$.

Proof. Let $J_{1}, J_{2}, J_{3}, \cdots$ denote an infinite sequence of closed curves satisfying the conditions of Theorem F. If $A_{n}, B_{n}, C_{n}$, and $D_{n}$ are four distinct points on $J_{n}$ in the order $A_{n} B_{n} C_{n} D_{n}$ then (Fig. 3) there exist on

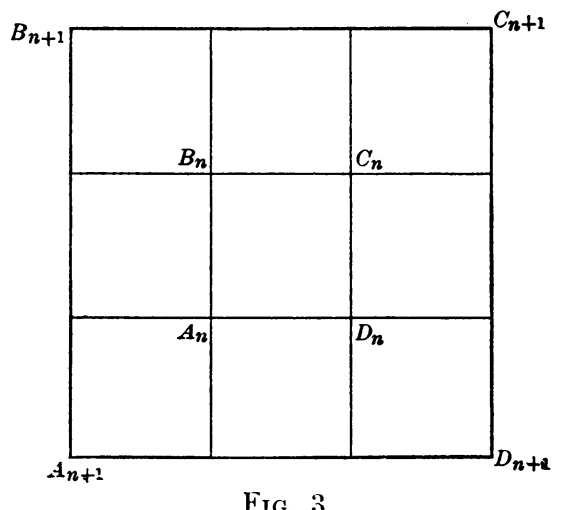

FIG. 3

$J_{n+1}$ four distinct points $A_{n+1}, B_{n+1}, C_{n+1}$, and $D_{n+1}$ such that $J_{n+1}$ can be divided by a double ruling, with two arcs parallel to $B_{n+1} C_{n+1}$ and $A_{n+1} D_{n+1}$ and the other two parallel to $A_{n+1} B_{n+1}$ and $C_{n+1} D_{n+1}$, into nine subdivisions of which the central one is $A_{n} B_{n} C_{n} D_{n} A_{n}\left(J_{n}\right)$. It follows that there exists a set of open curves* $\left[k_{m}\right](-\infty<m<\infty)$ and another set $\left[t_{n}\right](-\infty<n<\infty)$ such that (1) if $m$ and $n$ are any integers, positive or negative, $k_{m}$ has just one point in common with $t_{n}$, (2) for every integer $n$, the curve $t_{n}$ separates $t_{n-1}$ from $t_{n+1}$ and $k_{n}$ separates $k_{n-1}$ from $k_{n+1}$, (3) if $P$ is a point not lying on any open curve of either of these sets then there exist $m$ and $n$ such that $P$ is between $k_{m}$ and $k_{m+1}$ and also between $t_{n}$ and $t_{n+1}$. For every $m$ and $n$ let $A_{m n}$ denote the intersection of $k_{m}$ with $t_{n}$. Then the intervals $A_{m n} A_{m(n+1)}, A_{m(n+1)} A_{(m+1)(n+1)}, A_{(m+1)(n+1)} A_{(m+1) n}$, and $A_{(m+1) n} A_{m n}$ of the open curves $k_{m}, \dot{i}_{n+1}, k_{m+1}$, and $t_{n}$ respectively form a closed curve $J_{m n}$.

* Cf. Foundations, p. 159. 
By Theorem E there exist two sets of arcs $G_{m n}$ and $G_{m n}^{\prime}$ such that (1) each arc of $G_{m n}$ is entirely within $J_{m n}$ except that one of its endpoints is between $A_{m n}$ and $A_{(m+1) n}$ on the curve $t_{n}$ and the other one is between $A_{m(n+1)}$ and $A_{(m+1)(n+1)}$ on the curve $t_{n+1}$, (2) each arc of $G_{m n}^{\prime}$ is entirely within $J_{m n}$ except that one of its endpoints is between $A_{m n}$ and $A_{m(n+1)}$ on $k_{n}$ and the other one is between $A_{(m+1) n}$ and $A_{(m+1)(n+1)}$ on $k_{m+1}$, (3) through each point within $J_{m n}$ there is just one arc of the set $G_{m n}$ and just one arc of the set $G_{m n}^{\prime}$, (4) each point of $J_{m n}$, with the exception of $A_{m n}, A_{m(n+1)}, A_{(m+1) n}$, and $A_{(m+1)(n+1)}$, is an endpoint of just one arc of $G_{m n}$ or of just one arc of $G_{m n}^{\prime}$, (5) each arc of $G_{m n}$ has just one point in common with each arc of $G_{m n}^{\prime}$. If $P_{0}$ is a point on the arc $t_{0}$ and lying between the curves $k_{m}$ and $k_{m+1}, P_{0}$ is an endpoint of just one arc $g_{m 0}$ of the set $G_{m 0}$. The other endpoint of $g_{m 0}$ is a point $P_{1}$ lying between $k_{m}$ and $k_{m+1}$ on the curve $t_{1}$. The point $P_{1}$ is an endpoint of just one arc $g_{m 1}$ of the set $G_{m 1}$. The other endpoint of $g_{m 1}$ is a point $P_{2}$ lying between $k_{m}$ and $k_{m+1}$ on the curve $t_{2}$. This process may be continued. It follows that there exist two sequences of arcs $g_{m 0}, g_{m 1}, g_{m 2}, \cdots$ and $g_{m(-1)}, g_{m(-2)}, g_{m(-3)}$, ... such that (1) $P_{0}$ is an endpoint of $g_{m 0}$, (2) for every integer $n, g_{m n}$ is an arc of the set $G_{m n}$ and the arcs $g_{m n}$ and $g_{m(n+1)}$ have an endpoint in common. The sum of all the arcs $g_{m 0}, g_{m 1}, \cdots g_{m(-1)}, g_{m(-2)}, \cdots$ is clearly a continuous open curve, $l_{P_{0}}$, passing through the point $P_{0}$. Let $\alpha$ denote the set of all the curves $k_{m}$ for all integers $m$ together with the set of all the curves $l_{P}$ for all points $P$ on $t_{0}$. Similarly if $P$ is a point on $k_{0}$ there exists an open curve $r_{P}$ that passes through $P$ and is the sum of an infinite number of arcs each of which is, for some $m$ and $n$, an arc of the set $G_{m n}^{\prime}$. Let $\beta$ denote the set of all the curves $t_{n}$ for all intergers $n$ together with the set of all the curves $r_{P}$ for all points $P$ on $k_{0}$. It is clear that through every point there is just one curve of the set $\alpha$ and just one curve of the set $\beta$ and furthermore each curve of the set $\alpha$ has just one point in common with each curve of the set $\beta$.

Between the points of $\left\{\begin{array}{l}t_{0} \\ k_{0}\end{array}\right\}$ and the set of all real numbers there is a one-toone continuous correspondence $\left\{\begin{array}{l}\pi_{2} \\ \pi_{1}\end{array}\right\}$ in which the intersection of $k_{0}$ and $t_{0}$ is associated with the number 0 . If $P$ is any point let $\left\{\begin{array}{l}y_{P} P \\ x_{P}\end{array}\right\}$ denote the number which, in the correspondence $\left\{\begin{array}{l}\pi_{2} \\ \pi_{1}\end{array}\right\}$ is associated with the point in which $\left\{\begin{array}{l}t_{0} \\ k_{0}\end{array}\right\}$ intersects that curve of the set $\left\{\begin{array}{l}a \\ \beta\end{array}\right\}$ which passes through $P$. Thus to every point $P$ there corresponds a definite pair of real numbers $\left(x_{P}, y_{P}\right)$ and conversely.

It is clear that the correspondence thus established between $S$ and a number plane is continuous in the sense that in $S$ the point $P$ is a sequential limit point of the sequence of points $P_{1}, P_{2}, P_{3}, \cdots$ if, and only if, $\lim _{n=\infty} x_{P_{n}}=x_{P}$ and $\lim _{n=\infty} y_{P_{n}}=y_{P}$.

University of Pennsylvania 